DOI: $10.1515 /$ biolog-2016-0117

Due to a misunderstanding caused by the author, Dr. Shamik Dasgupta, the name of the co-author, Dr. Jiasong Fang (who has been acknowledged in the manuscript), was omitted in the original publication. The correct authorship of this article is below.

REVIEW

\title{
Mitochondrial clock: moderating evolution of early eukaryotes in light of the Proterozoic oceans
}

\author{
Shamik Dasgupta ${ }^{1} \&$ Jiasong FAnG $^{2,3, *}$ \\ ${ }^{1}$ Deep Sea Science Division, Institute of Deep-Sea Science and Engineering, Chinese Academy of Sciences, Sanya, 572000 \\ Hainan, People's Republic of China \\ ${ }^{2}$ The Hadal Science and Technology Research Center, Shanghai Ocean University, 999 Huchenghuan Road, Shanghai 201306, \\ China \\ ${ }^{3}$ College of Natural and Computational Sciences, Hawaii Pacific University, Kaneohe, HI 96744, USA; e-mail: \\ jfang@hpu.edu
}

\footnotetext{
* Corresponding author 